\title{
Rooted trees and moments of large sparse random matrices
}

\author{
Oleksiy Khorunzhiy
}

Université de Versailles Saint-Quentin, Versaiiles 78035, France

khorunjy@math.uvsq. fr

In these expository paper we describe the role of the rooted trees as a base for convenient tools in studies of random matrices. Regarding the Wigner ensemble of random matrices, we represent main ingredients of this approach. Also we refine our previous result on the limit of the spectral norm of adjacency matrix of large random graphs.

Keywords: Random matrices, spectral norm, rooted trees

\section{Introduction}

In late 50th $\mathrm{E}$. Wigner studied the moments of $N \times N$ random symmetric matrices whose entries are independent identically distributed real random variables [Wig55]. He observed that if the law of these variables is symmetric, then after certain normalization, the leading contribution to the $2 k$-th moment $M_{2 k}^{(N)}$ as $N \rightarrow \infty$ is described by the set of simple random walks of $2 k$ steps in the upper half-plane starting and ending at zero. Moreover, the $\operatorname{limit}_{\lim _{N \rightarrow \infty}} M_{2 k}^{(N)}$ is proportional to the number of these walks.

Later this description was combined with the graph theory tools to study the spectral norm of large random matrices of this class known as the Wigner ensemble [BY88, FK81]. The use of the graph theory is possible here due to the one-to-one correspondence between the simple half-plane random walks and the set of rooted trees $T_{k}$ with $k$ edges drawn in the upper half-plane. Another version of the random walks representation is used to prove the universal character of extreme eigenvalue statistics of large random matrices of the Wigner ensemble [Sos99].

The common feature of these works is that one considers the moments $M_{2 k}^{(N)}$ in the limit when $k$ increases proportionally to some power of $N$ [Gem]. Then one has to take into account not only the leading contribution to $M_{2 k}^{(N)}$, but also next terms of $1 / N$-expansion of it. To do this, modifications of the method of [Wig55] were proposed [BY88, FK81, Sos99] that involve additional combinatorial constructions.

In paper [Kho01] it was shown that the trees still represent a simple and convenient description of the corrections to $M_{2 k}^{(N)}$. Namely, it was proved that the rooted trees added by the procedure of vertex gluing and shift of cycles describe all terms of $1 / N$-expansion of $M_{2 k}^{(N)}$. On this way one can separate two different classes of graphs obtained from trees:

(A) those that have gluings of children with different parents and

(B) those that glue the children of the same parent. 
This gives a natural and simple description of two types of corrections to $M_{2 k}^{(N)}$ observed independently in [Sos99]. In the Wigner ensemble, the corrections (A) contribute more than those of (B) and [Sos99] is mostly related with the corrections of the first type.

Corrections (B) become crucial in the ensemble of sparse random matrices related to adjacency matrix of random graphs. The ensemble consists of $N \times N$ matrices $H^{(N, p)}$ that have, in average, $p$ non-zero elements per row. In paper [Kho01] it is proved that if $p=O\left((\log N)^{1+\delta}\right)$ with $\delta>0$, then the spectral norm of $\frac{1}{\sqrt{p}} H^{(N, p)}$ converges to $2 v$ as $N \rightarrow \infty$. In present paper we show that condition $p(\log N)^{-1} \rightarrow \infty$ is sufficient for this convergence. The proof uses the properties of the set of rooted trees. We present refinements of results of [Kho01] on this subject also.

\section{Moments of Wigner random matrices}

To model the energy levels of heavy atomic nuclei, E. Wigner proposed to consider the eigenvalues of real symmetric $N \times N$ matrices $A^{(N)}$

$$
\left[A^{(N)}\right]_{i j}=\frac{1}{\sqrt{N}} a_{i j}, \quad i, j=1, \ldots, N
$$

where $\left\{a_{i j}, i \leq j\right\}$ are identically distributed random variables with zero mean value and variance $v^{2}$. He showed that if all even moments of $a_{i j}$ are bounded and all odd moments of $a_{i j}$ vanish, then the normalized traces of $A^{(N)}$ converge in average as $N \rightarrow \infty$;

$$
\lim _{N \rightarrow \infty} \mathbf{E}\left\{\frac{1}{N} \operatorname{Tr}\left[A^{(N)}\right]^{l}\right\}=m_{l}= \begin{cases}v^{2 k} t_{k}, & \text { if } l=2 k \\ 0, & \text { if } l=2 k+1,\end{cases}
$$

where $t_{k}$ are the well-known Catalan numbers determined by recurrent relations

$$
t_{0}=1, \quad t_{k}=\sum_{j=0}^{k-1} t_{k-1-j} t_{j},
$$

and are given by equality $t_{k}=\frac{1}{k+1}\left(\begin{array}{c}2 k \\ k\end{array}\right)$. The limiting moments $m_{l}$ determine the semicircle density $\rho(\lambda)$ :

$$
m_{l}=\int_{-\infty}^{\infty} \lambda^{l} \rho(\lambda) \mathrm{d} \lambda=\left(2 \pi v^{2}\right)^{-1} \int_{-2 v}^{2 v} \lambda^{l} \sqrt{4 v^{2}-\lambda^{2}} \mathrm{~d} \lambda
$$

and convergence (2) is called the semicircle (or Wigner) law for the eigenvalue counting function $\sigma\left(\lambda ; A^{(N)}\right)=$ $\frac{1}{N} \#\left\{\lambda_{j}^{(N)} \leq \lambda, 1 \leq j \leq N\right\}$. It implies the weak convergence of the measures

$$
\mathrm{d} \sigma\left(\lambda ; A^{(N)}\right) \rightarrow \rho(\lambda) \mathrm{d} \lambda, \quad N \rightarrow \infty .
$$

Later it was proved that this convergence holds with probability 1.

Let us briefly explain why the Catalan numbers $t_{k}$ arise in the study of the moments

$$
\mathbf{E}\left\{\frac{1}{N} \operatorname{Tr}\left[A^{(N)}\right]^{2 k}\right\}=\frac{1}{N^{k+1}} \sum_{i} \mathbf{E} a_{i_{1} i_{2}} a_{i_{2} i_{3}} \cdots a_{i_{2 k} i_{1}} .
$$



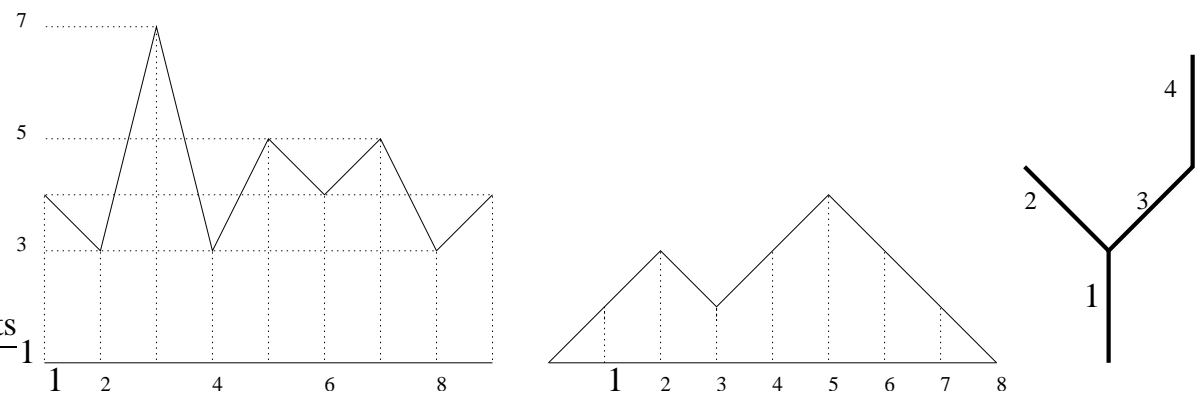

Fig. 1: Set $I_{8}^{\prime}=(4,3, \ldots, 4)$, corresponding random walk and tree $\tau$.

The last mathematical expectation is nonzero only for those sets (or walks) $I_{2 k}=\left(i_{1}, i_{2}, \ldots, i_{2 k}, i_{1}\right)$ where the random variables $a$ are present even number of times.

The random variables $a_{i_{r} i_{r+1}}$ are labelled by the steps of the walk $I_{2 k}$. Thus one takes into account the walks $I^{\prime}$, where each step $(\alpha, \beta)$ is passed an even number of times, when regarded in both directions. It is easy to see that the leading contribution to (4), in the limit $N \rightarrow \infty$, is given by the walks $I_{2 k}^{\prime}$ where each step is passed two times only: there and back (see figure 1). The set of these walks $I_{2 k}^{\prime}$ is separated into classes of equivalence, and these classes are labelled by half-plane rooted trees $\tau \in T_{k}$ of $k$ edges. The number of classes $t_{k}=\left|T_{k}\right|$ is the Catalan number (3). This is the way E. Wigner has counted the limiting moments $m_{l}$ [Wig55].

One can restore $I_{2 k}^{\prime}$ from $T_{k}$ as follows: take $\tau$ and assign to vertices of $\tau$ different numbers from the set $(1, \ldots, N)$. The lexicographical path over edges of $\tau$ that starts and ends at the root produce a walk of the type $I_{2 k}^{\prime}$. There are $N(N-1) \cdots(N-k)=O\left(N^{k+1}\right)$ walks of the type $I_{2 k}^{\prime}$ corresponding to $\tau$.

\section{Two types of corrections to the semicircle law}

According to the arguments of [Wig55] presented above, relation (4) can be rewritten in the form

$$
M_{2 k}^{(N)}=\mathbf{E}\left\{\frac{1}{N} \operatorname{Tr}\left[A^{(N)}\right]^{2 k}\right\}=v^{2 k} t_{k}+R_{K}^{(N)}, \quad R_{k}^{(N)}=O\left(\frac{Q_{k}}{N}\right) .
$$

The factor $\left(v^{2}\right)^{k}$ arises due to the fact that in $I_{2 k}$ corresponding to trees $\tau$ each step is passed exactly two times.

The situation is different for the terms $Q_{k}$. First, there are terms that still have powers of $v^{2}$ only; second, there are terms where the higher moments $V_{2 s}=\mathbf{E} a^{2 s}$ are present. The walks corresponding to the first type of terms can be described by graphs obtained from the trees after the gluings of children of different parents (see figure 2).

In the second case the gluings are made between children of the same parents (see figure 3 ). These produce the factors $V_{2 s}, s \geq 2$. This difference plays an important role in the universal behaviour of the edge spectral statistics of Wigner ensemble. Loosely speaking, the corrections of the second type vanish 
148

Oleksiy Khorunzhiy
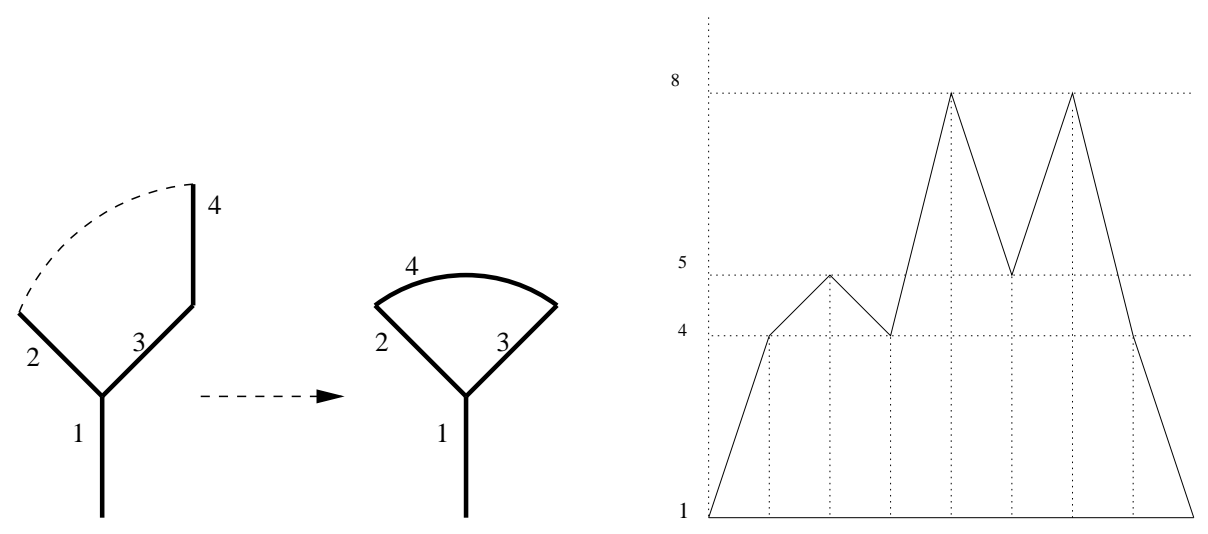

Fig. 2: Gluings of the first type and corresponding walk
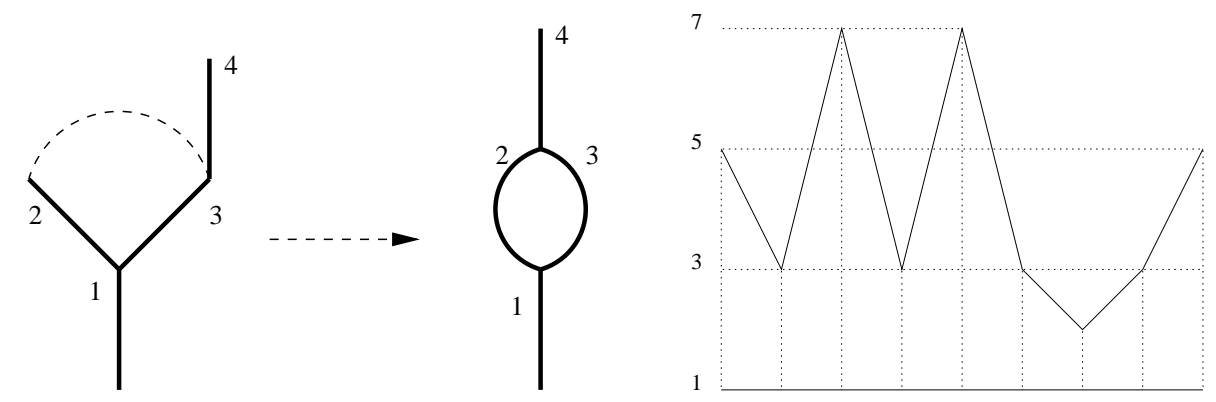

Fig. 3: Gluing of the second type and corresponding walk 
from $M_{2 k}^{(N)}$ faster than those of the first type in the limit $N, k \rightarrow \infty$. That is why the asymptotic expression for $M_{2 k}^{(N)}$ does not contain the values $V_{2 k}$ [Sos99].

Corrections to $M_{2 k}^{(N)}$ determine the limit of the spectral norm $\left\|A^{(N)}\right\|=\lambda_{\max }^{(N)}=\max _{j=1, \ldots, N}\left|\lambda_{j}^{(N)}\right|($ see [BY88, FK81, Gem]). Indeed, elementary inequality

$$
P_{N}(u)=\operatorname{Prob}\left\{\lambda_{\max }^{(N)} \geq u\right\} \leq \frac{1}{u^{k}} N M_{2 k}^{(N)}
$$

shows that if one can prove the estimate $M_{2 k}^{(N)} \leq v^{2 k} t_{k}(1+\varepsilon / 2)^{2 k}$ with $\varepsilon>0$ for all $k=O(\log N), N \rightarrow \infty$, then one can conclude that

$$
\limsup _{N \rightarrow \infty} \lambda_{\max }^{(N)} \leq 2 v
$$

with probability 1 . This observation together with the convergence of $\sigma\left(\lambda ; A^{(N)}\right)$ implies convergence $\lambda_{\max }^{(N)} \rightarrow 2 v$ with probability 1 as $N \rightarrow \infty[\mathrm{BY} 88, \mathrm{FK} 81, \mathrm{Gem}]$.

\section{Moments of sparse random matrices}

Let us consider the ensemble of random real symmetric matrices

$$
\left[H^{(N, p)}\right]_{i j}=d_{i j} a_{i j}
$$

where the random variables $\left\{d_{i j}, i \leq j\right\}$ of the law

$$
d_{i j}=\frac{1}{\sqrt{p}} \begin{cases}1, & \text { with probability } p / N, \\ 0, & \text { with probability } 1-p / N\end{cases}
$$

are independent between themselves and independent of $a_{i j}$. In the limit $p / N \rightarrow 0$ matrices (7) become very sparse and the ensemble is statistically different from the Wigner ensemble (1). The aim of this paper is to prove the following statement.

Theorem 1. Let random variables $a_{i j}$ verify condition $\mathbf{E}\left(a_{i j}\right)^{2 k} \leq(C k)^{k}$ with some $C>0$. If $p_{N}(\log N)^{-1} \rightarrow$ $\infty$ as $N \rightarrow \infty$, then the spectral norm of (7) is bounded with probability 1 ;

$$
\limsup _{N \rightarrow \infty} \lambda_{\max }^{\left(N, p_{N}\right)} \leq 2 v
$$

Remark. In paper [Kho01] this bound is proved in the limit $N \rightarrow \infty$ and $p_{N}=(\log N)^{1+\delta}, \delta>0$.

The proof of Theorem 1 is based on (6) and therefore involves the study of high moments of (7). The leading term of the moment

$$
M_{2 k}^{(N, p)}=\mathbf{E}\left\{\frac{1}{N} \operatorname{Tr}\left[H^{(N, p)}\right]^{2 k}\right\}
$$

is still described by the Catalan numbers as in (5), but the term $R_{k}^{(N)}$ is replaced by the sum of the terms of the order $O\left(p^{-l} N^{-m}\right), l+m \geq 1$. The terms of the order $p^{-l}$ correspond to gluings of the children of the same parent, the factors $N^{-m}$ arise due to gluings of children of different parents. 
In paper [Kho01] it is shown that if $V_{2 s} \leq(C s)^{s}$, then

$$
M_{2 k}^{(N, p)} \leq v^{2 k} t_{k}+\sum_{s=1}^{k} \frac{C^{s}}{(2 p)^{s}} \frac{1}{s !} \sum_{\tau \in T_{k}}\left(b_{1}^{2}+b_{2}^{2}+\ldots+b_{k+1}^{2}\right)^{s}+O\left(k^{2} N^{-1}\right)
$$

where $b_{j}=b_{j}(\tau) \geq 0$ is equal to the number of children of the vertex $j$ of $\tau$ and $b_{1}+\ldots+b_{k+1}=k$. Obvious inequality

$$
\frac{1}{s !} \sum_{\tau \in T_{k}}\left(b_{1}^{2}+b_{2}^{2}+\ldots+b_{k+1}^{2}\right)^{s} \leq \frac{t_{k} k^{s}}{s !} \frac{1}{t_{k}} \sum_{\tau \in T_{k}} B_{\tau}^{s}, \quad B_{\tau}=\max _{j=1, \ldots, k} b_{j}
$$

shows that the distribution of the maximal degree of equiprobable trees is essential for estimates of $M_{2 k}^{(N, p)}$.

\section{Trees, vertices, children}

The following proposition improves observations of [Kho01].

Lemma 1. Denote by $T_{k}^{(b)}$ the set of trees $\tau^{\prime} \in T_{k}$ such that $B_{\tau^{\prime}}=b \leq k$. Then

$$
t_{k}(b)=\left|T_{k}^{(b)}\right| \leq k t_{k}\left(1-D_{b-2}\right)^{b-4}
$$

where $D_{b}>1 / 4$ and $\lim _{b \rightarrow \infty} D_{b}=1 / 2$.

Remark. In paper [Kho01] inequality (11) is proved with $D_{b}=1 / 4$.

To prove Lemma 1 , let us estimate the number $\hat{t}_{k}^{(b)}$ of trees $\tau^{\prime \prime} \in T_{k}$ such that the degree of the root of $\tau^{\prime \prime}$ is equal to $b$. One can consider $\hat{t}_{k}^{(b)}$ also as the number of forests constructed on $b$ roots with the help of $k-b$ edges. We denote this number by $n_{k-b}^{(b)}$.

Lemma 2. Given $b \geq 2$, the following estimate is true

$$
n_{k-b}^{(b)}=\hat{t}_{k}^{(b)} \leq\left(1-D_{b}\right)^{b-2} t_{k-1}
$$

Proof. More general form of (3)

$$
\hat{t}_{k}^{(b)}=\sum_{j=0}^{k-b} \hat{t}_{k-1-j}^{(b-1)} t_{j}
$$

implies inequality $\hat{t}_{k}^{(b)} \leq t_{k-1}$. Then we deduce from (13) that

$$
\hat{t}_{k}^{(b)} \leq \sum_{j=0}^{k-b} t_{k-2-j} t_{j}=t_{k-1}-\left(t_{0} t_{k-2}+\ldots+t_{b-1} t_{k-1-b}\right) .
$$

It is easy to see that $t_{k-2}=\frac{1}{k-1}\left(\begin{array}{c}2 k-2 \\ k-1\end{array}\right) \geq \frac{1}{4} t_{k-1}$. Then

$$
t_{0} t_{k-2}+\ldots+t_{b-1} t_{k-1-b} \geq \frac{t_{k-1}}{4} \sum_{l=0}^{b} \frac{t_{l}}{4^{l}} \equiv D_{b} t_{k-1},
$$


where $D_{b}$ tends to $4^{-1} f\left(4^{-1}\right)=1 / 2, f$ being the generating function of the Catalan numbers.

Now we can conclude that if $b \geq 2$, then $\hat{t}_{k}^{(b)} \leq\left(1-D_{b}\right) t_{k-1}$. Regarding a sequence of relations (13) with $b=2,3, \ldots$, we obtain the estimate (12). Finally, more general than (13) relation

$$
n_{s-l}^{(r+l)}=\sum_{j=0}^{s-l} n_{j}^{(r)} n_{s-l-j}^{(l)},
$$

when combined with (12), implies inequality

$$
n_{s-l}^{(r+l)} \leq\left(1-D_{l}\right)^{l-2} n_{s-1}^{(r+1)}, \quad 2 \leq l \leq s .
$$

Lemma 2 is proved.

Proof of Lemma 1. To accomplish this on the base of (14), we introduce the following description of the set $T_{k}$. Let us consider $k$ numerated edges, take first $h$ of them and construct all trees $T_{h-1}, 1 \leq h \leq k$.

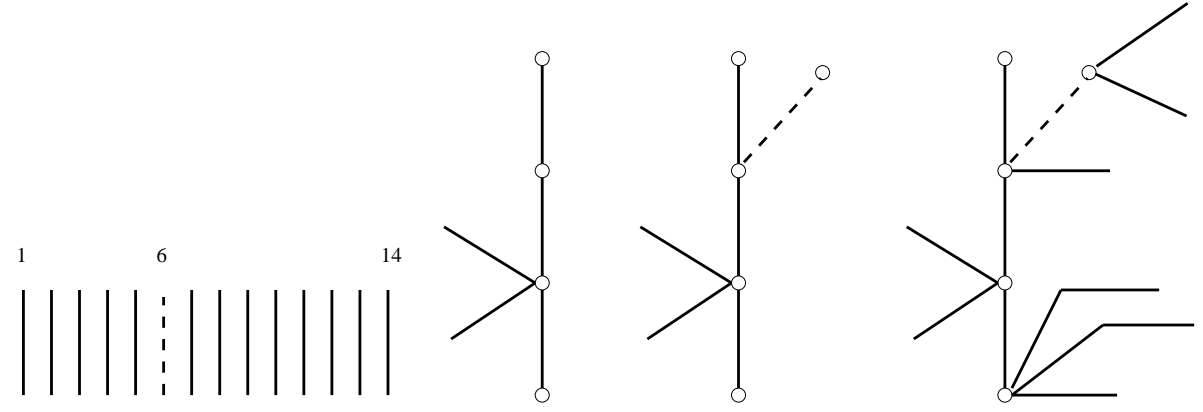

Fig. 4: Construction of trees of $T_{14}$ with $\alpha=3, \beta=2$ and $h=6$.

We can do this in agreement with the lexicographical order: regarding already constructed tree $\tau \in T_{j}$, we attach the edge number $j+1$ to one of the vertices of the border of $\tau_{j}$. We call the border of the tree $\tau \in T_{j}$ the set of its vertices such that the new edge adjacent to one of these vertices will be the last in the lexicographical order (see figure 4 , where the border of the tree is shown by small circles).

Given $h \geq 2$, let us consider a tree $\tau$ of $T_{h-1}$ that have the border of $\alpha+1$ vertices, $1 \leq \alpha \leq h-1$. Then the edge $h$ can be attached to one of the $\alpha$ vertices the most closes to the root. Let us denote by $\beta$ the position of this vertex in the border of $\tau$. There remains $k-h$ edges in our disposition; we use them to construct the forest on $\beta+1$ roots. The resulting tree belongs to $T_{k}$ and all of these trees can be obtained on this way by varying $\alpha$ and $\beta$. Therefore,

$$
t_{k}=\sum_{\alpha=1}^{h-1} n_{h-1-\alpha}^{(\alpha)} \sum_{\beta=1}^{\alpha+1} n_{k-h}^{(\beta+1)} .
$$

In this relation we assume $h>1$. The case of $h=1$ can also be included under agreement that $\alpha=0$ and $n_{0}^{(0)}=1$.

Now let us construct the set of $k$-edge trees $\tilde{T}_{k}^{(b)}$ that have at least one vertex with the number of children equal to $b$. 


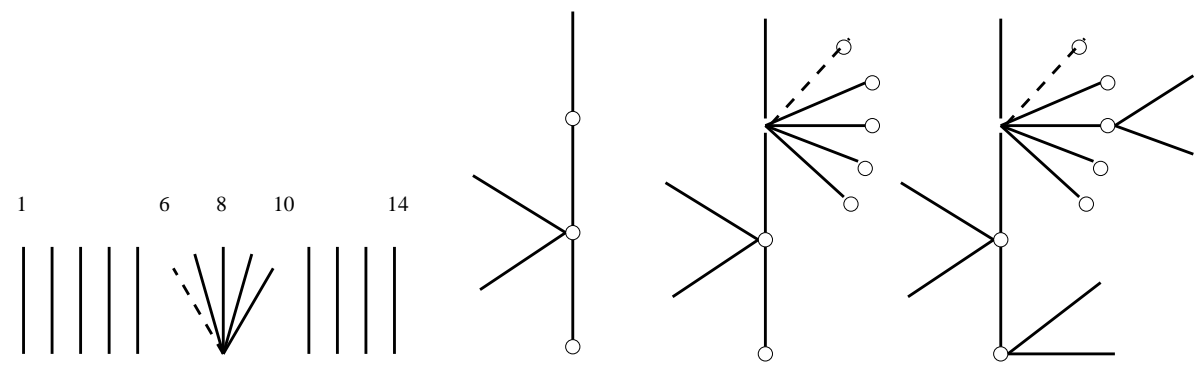

Fig. 5: Construction of trees of $T_{14}$ with $h=6$ and $b-2=6$.

To do this, we modify the procedure described above, assuming that the edge number $h$ and the next $b-2$ edges are considered as one cluster, i.e. the set of $b-1$ children of one parent (see figure 5).

Then one can write equality

$$
\left|\tilde{T}_{k}^{(b)}\right|=\sum_{h=1}^{k-b+2} \sum_{\alpha=1}^{h-1} n_{h-1-\alpha}^{(\alpha-1)} \sum_{\beta=1}^{\alpha} n_{k-h-b+2}^{(\beta+b-2)} .
$$

This relation taken with $b=2$ differs from (15) for the reason that in (16) we have prohibited to attach the edges to the vertex, where the edge $h$ is attached; also we do not attach the edge $h$ to the vertex $\alpha+1$ of the border of the tree. Certainly, these restrictions diminish the number of trees obtained. Now, applying (14) to the last factor of (16), we obtain inequalities

$$
\left|\tilde{T}_{k}^{(b)}\right| \leq \sum_{h=1}^{k-b+2} \sum_{\alpha=1}^{h-1} n_{h-1-\alpha}^{(\alpha-1)} \sum_{\beta=1}^{\alpha} n_{k-h}^{(\beta)}\left(1-D_{b-2}\right)^{b-4} \leq k t_{k-1}\left(1-D_{b-2}\right)^{b-4}
$$

Obvious inclusion $T_{k}^{(b)} \subseteq \tilde{T}_{k}^{(b)}$ completes the proof of (11).

\section{Proof of Theorem 1}

Let us continue inequalities (9) and (10). We chose $B_{0}>4$ such that $\chi=\chi_{B_{0}}=\ln \left(1-D_{B_{0}}\right)<1$. Then

$$
\begin{gathered}
\frac{1}{s ! t_{k}} \sum_{\tau \in T_{k}} B_{\tau}^{s} \leq \frac{B_{0}^{s}}{s !}+\frac{1}{s ! t_{k}} \sum_{j=0}^{k-B_{0}-1} \sum_{\left\{\tau: B_{0}+j<B_{\tau} \leq B_{0}+j+1\right\}} B_{\tau}^{s} \leq \\
B_{0}^{s}\left\{1+\frac{1}{s !} k\left(1-D_{\left.B_{0}+j-2\right)^{B_{0}-4}} \sum_{j=0}^{\infty}(1+j)^{s} e^{-j \ln \left(1-D_{B_{0}+j-2}\right)}\right\} \leq\right. \\
B_{0}^{s}\left\{1+2 k e^{\chi} e^{-\left(B_{0}-4\right) \chi} \chi^{s+1}\right\} \leq B_{0}^{s}\left(1+6 k e^{-\left(B_{0}-4\right)}\right) .
\end{gathered}
$$

Substituting this estimate into the right-hand side of (9), we obtain inequality

$$
M_{2 k}^{(N, p)} \leq v^{2 k} t_{k}\left[1+\sum_{s=1}^{k}\left(\frac{C k B_{0}}{2 p}\right)^{s}\left\{1+6 k e^{-\left(B_{0}-4\right) \chi}\right\}\right] .
$$


It follows from this inequality that if $p=C k B_{0} / 2$, then

$$
M_{2 k}^{(N, p)} \leq 2(k+1)^{2} v^{2 k} t_{k}
$$

Using this estimate and inequality (6), we complete the proof of Theorem 1 . Indeed, given $\varepsilon>0$, we choose $k=(2+\Delta) \ln N[\ln (1+\varepsilon)]^{-1}$ with $\Delta>0$ and for sufficiently large $N, p$ becomes larger than $C B_{0} k$ and (17) holds. Then

$$
\operatorname{Prob}\left\{\lambda_{\max }^{(N)} \geq 2 v(1+\varepsilon)\right\} \leq \frac{N M_{2 k}^{(N)}}{(2 v)^{2 k}(1+\varepsilon)^{2 k}} \leq \frac{2(k+1)^{2} N}{(1+\varepsilon)^{2 k}} \leq \frac{(4+\Delta)^{2}[\ln N]^{2}}{N^{1+\Delta}} .
$$

Therefore the series $\sum_{N} P_{N}(2 v(1+\varepsilon))$ converges and the Borel-Cantelli lemma implies the upper bound

$$
\limsup _{N \rightarrow \infty} \lambda_{\max }^{\left(N, p_{N}\right)} \leq 2 v(1+\varepsilon) \quad \text { with probability } 1 .
$$

This completes the proof of Theorem 1.

\section{Discussion}

Let us consider a particular case of $H^{(N, p)}(7)$ with Bernoulli random variables $a_{i j}= \pm 1$ with probabilities $1 / 2$ and study random variables

$$
\eta^{(N, p)}=\max _{j=1, \ldots, N}\left\|H^{(N, p)} e^{(j)}\right\|^{2}
$$

where $e^{(j)}(x)=\delta_{x, j}$. It is easy to show that if $p_{N}(\log N)^{-1} \rightarrow 0$, then with probability 1

$$
\lim _{N \rightarrow \infty} \eta^{\left(N, p_{N}\right)}>r \text { for any } r>0
$$

This means that the value $p_{N}^{\prime}=\log N$ is critical for the spectral norm of $H^{(N, p)}$ to be bounded or not.

Random variable (19) is closely related with the maximum of partial sums of the Erdôs-Rényi limit theorem [Kho02]. In this connection it would be interested to analyse the spectral norm of $H^{(N, p)}$ in the limit $p=\tau \log N$. On can conject that the spectral norm will depend both on the value of $\tau$ and the function $\phi(t)=\mathbf{E} \exp \left\{t a_{i j}\right\}$. In this studies the detailed analysis of the multigraphs obtained from trees should be carried out.

Let us also note that if $p \rightarrow \infty$, then $\mathrm{d} \sigma\left(\lambda ; H^{(N, p)}\right)$ converges in probability to the semicircle distribution $\rho(\lambda) \mathrm{d} \lambda$ (see e.g. [AKS92, RB88]). This can be proved by using the resolvent technique [AKS92] as well as regarding the moments $M_{2 k}^{(N, p)}$ (9) with fixed $k$ and $p \rightarrow \infty$.s Then one can say that in the limit $1<<p<<\log N$ the upper bound of the support of $\sigma\left(\lambda ; H^{(N, p)}\right)$ is bounded, but some of eigenvalues go to infinity.

In papers [FK81, KS03] the maximal eigenvalue of the adjacency matrix of the random graph is studied. The matrix is given by $\left[D^{(N, p)}\right]_{i j}=\sqrt{p} d_{i j}$ (8). The difference between $D^{(N, p)}$ and $H^{(N, p)}$ is that in our case the random matrix entries have zero mathematical expectation because of the weights $a_{i j}$. Also normalization by $\sqrt{p}$ justified by theoretical physics studies makes results of [KS03] different from ours.

Another spectral characteristics is the diffusion coefficient of the system corresponding to random matrices $H^{(N, p)}$. Here another critical value $p^{\prime \prime}$ appears that separates two states of localized and delocalized 
eigenvectors. Numerical and theoretical physics arguments show that $p^{\prime \prime}$ is finite [Eva83, MF91]. For this

case of finite parameter $p$, closed recurrent relations that determine $m_{2 k}^{(p)}=\lim _{N \rightarrow \infty} M_{2 k}^{(N, p)}$ are obtained using the trees and walks with repetitions over them $[\mathrm{BG} 01, \mathrm{KV}]$. It would be interesting to study the diffusion coefficient of $H^{(N, p)}$ using the approach developed.

\section{References}

[AKS92] L. Pastur A. Khorunzhy, B. Khoruzhenko and M. Shcherbina. The large-n limit in statistical mechanics and spectral theory of disordered systems. Phase Transitions and Critical Phenomena, 15:73-239, 1992.

[BG01] M. Bauer and O. Golinelli. Random incidence matrices: moments of the spectral density. $J$. Stat. Phys., 103:301-337, 2001.

[BY88] Z.D. Bai and Y.Q. Yin. Necessary and sufficient conditions for almost sure convergence of the largest eigenvalue of a wigner matrix. Ann. Probab., 16:1729-1741, 1988.

[Eva83] S.N. Evangelou. Quantum percolation and the anderson transition in dilute systems. J. Stat. Phys., 27:1397-1400, 1983.

[FK81] Z. Füredi and J. Komlós. The eigenvalues of random symmetric matrices. Combinatorica, $1: 233-241,1981$.

[Gem] S. Geman. A limit theorem for the norm of random matrices.

[Kho01] A. Khorunzhy. Sparse random matrices: spectral edge and statistics of rooted trees. Adv. Appl. Probab., 33:124-140, 2001.

[Kho02] A. Khorunzhy. Stochastic version of the erdős-rényi limit theorem. Probab. Mathem. Statistics, 22:221-230, 2002.

[KS03] M. Krivelevich and B. Sudakov. The largest eigenvalue of sparse random graphs. Combin. Probab. Comput., 12:61-72, 2003.

[KV] A. Khorunzhy and V. Vengerovsky. On asymptotic solvability of random graph's laplacians. Preprint available at http://xxx.lanl.gov math-ph/0009028.

[MF91] A.D. Mirlin and Ya. V. Fyodorov. Universality of level correlation function of a sparse random matrix. J. Phys. A, 24:2273-2286, 1991.

[RB88] G.J. Rodgers and A.J. Bray. Density of states of sparse random matrix. Phys. Rev. B, 37:3557$3562,1988$.

[Sos99] A. Soshnikov. Universality at the edge of the spectrum in wigner random matrices. Commun. Math. Phys., 207:697-733, 1999.

[Wig55] E. Wigner. Characteristic vectors of bordered matrices with infinite dimensions. Ann. Math., 62:548-564, 1955. 